\title{
Rhabdomyosarcoma of the Paranasal Sinuses Initially Diagnosed as Acute Sinusitis
}

\author{
Amanda E. Dilger ${ }^{1, *}$, Alexander L. Schneider ${ }^{1}$, John Cramer $^{1}$ and Stephanie Shintani Smith ${ }^{1,2}$ \\ 1 Department of Otolaryngology-Head and Neck Surgery, Northwestern University Feinberg School of \\ Medicine, Chicago, IL 60611, USA; alexander.schneider@northwestern.edu (A.L.S.); \\ john.cramer@northwestern.edu (J.C.); sshintani@gmail.com (S.S.S.) \\ 2 Center for Healthcare Studies, Northwestern University Feinberg School of Medicine, \\ Chicago, IL 60611, USA \\ * Correspondence: amanda.dilger@northwestern.edu
}

Academic Editor: César Picado

Received: 14 November 2016; Accepted: 16 January 2017; Published: 4 February 2017

\begin{abstract}
Rhabdomyosarcoma (RMS) is an uncommon soft tissue malignancy that is typically found in the pediatric population. Here we describe a rare case of widely metastatic alveolar RMS of the right paranasal sinuses in an adult woman who presented with several months of unilateral sinus symptoms that was initially misdiagnosed as acute sinusitis. A middle-aged female presented with two months of right sinus pressure and unilateral epistaxis. She had previously been diagnosed with acute sinusitis and was treated with antibiotics without improvement. Nasal endoscopy demonstrated a fungating right nasal cavity mass. On computed tomography scan (CT), she was found to have metastatic disease in the mediastinum, lungs, bones, pancreas, and right ovary. Pathology of the nasal cavity mass was consistent with alveolar RMS. The patient initially responded well to chemotherapy, but subsequently developed brain and leptomeningeal metastases. This case of sinonasal rhabdomyosarcoma is unique in the extent of metastatic disease at the time of diagnosis and the initial misdiagnosis despite concerning unilateral symptoms and imaging. This thus highlights the importance of maintaining a high index of suspicion for malignancy in patients with unilateral sinus symptoms.
\end{abstract}

Keywords: unilateral; sinusitis; rhabdomyosarcoma

\section{Introduction}

Soft tissue sarcomas are rarely occurring heterogeneous tumors derived from embryonic mesoderm [1]. They are primarily pediatric malignancies, having been shown to comprise up to $12 \%$ of pediatric malignancies, but as little as $1 \%$ of all adult malignancies [2]. Sarcomas account for only $1 \%$ to $2 \%$ of all head and neck malignancy [3-5]. The rhabdomyosarcoma (RMS) subtype is even more rare in adults, representing less than $1 \%$ of all malignant solid tumors in adults. When it does occur, one of the most common RMS subsites is the head and neck region [6].

Within the head and neck, RMS is further sub-divided into three main sub-types: orbital, nonorbital parameningeal (including the sinonasal region), and nonorbital nonparameningeal. RMS of the head and neck often produces non-specific symptoms secondary to local mass effect, including headache, nasal congestion, and otorrhea, all of which mimic benign disease. Thus, it is often diagnosed late in its course. Parameningeal sites confer a significant risk of central nervous system dissemination, and unfortunately are often unresectable by the time of diagnosis [7]. It is thus not surprising that parameningeal RMS are associated with a poor prognosis, and are at high risk of early recurrence [8]. 
Here, we describe a rare case of widely metastatic alveolar rhabdomyosarcoma of the paranasal sinus in an adult woman who presented with several months of unilateral sinus and ophthalmologic symptoms.

\section{Case Report}

A previously healthy middle-aged woman initially presented to an outside hospital Emergency Room with the complaint of headache and right sided sinus/facial pressure. Computed tomography scan (CT) brain at that time showed complete opacification of the right maxillary, ethmoid, and sphenoid sinuses (Figure 1). She was presumptively diagnosed with acute on chronic sinusitis and prescribed a course of oral antibiotics, oral steroids, and intra-nasal steroids. Approximately two weeks after this presentation, she had a breast biopsy for a newly noted mass, the pathology of which was consistent with poorly differentiated malignant neoplasm.

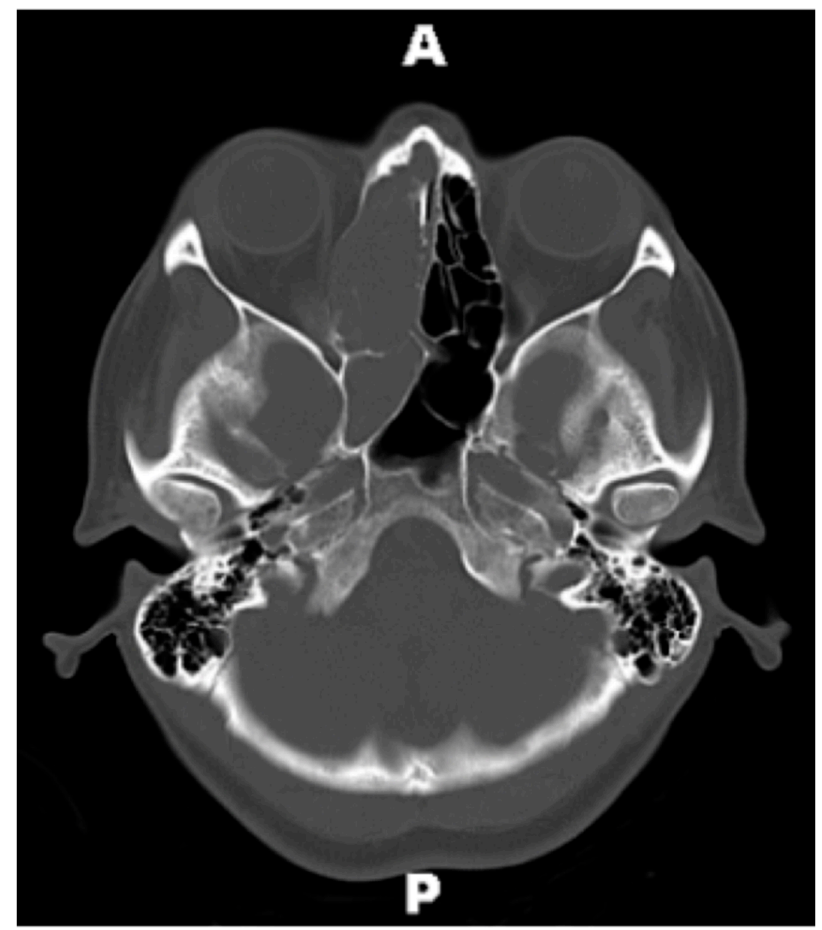

Figure 1. Prehospitalization computed tomography scan (CT) Brain without Contrast, axial view. Initial CT obtained by primary care physician, which demonstrates complete opacification of the right ethmoid and sphenoid sinuses. Abbreviations: $\mathrm{A}=$ anterior; $\mathrm{P}=$ posterior.

Three months after her initial presentation, she re-presented to the Emergency Department with right-sided epistaxis, hemoptysis, shortness of breath, and worsening right sinus pressure. Physical exam was notable for right-sided exophthalmos and epiphora with diplopia on far lateral gaze. Cranial nerve exam demonstrated paresthesias in the right V2 distribution. An otolaryngologist was consulted and nasal endoscopy revealed a fungating, bleeding mass that appeared to be originating from the head of the inferior turbinate and obstructing the entire right nasal cavity. The left nasal cavity appeared normal. Routine laboratory tests were notable for thrombocytopenia with platelets of 49,000.

CT Neck/Sinus demonstrated a large, enhancing mass in the right nasal cavity measuring $5.9 \mathrm{~cm} \times 3.7 \mathrm{~cm} \times 5.3 \mathrm{~cm}$, which involved the entire right ethmoid sinuses and right maxillary sinus with post-obstructive opacification of the right frontal and sphenoid sinuses (Figure 2). There was also extension into the right orbit, with mass effect on the medial rectus and optic nerve as well extension into the right anterior cranial fossa. Multiple enlarged retropharyngeal, supraclavicular, and mediastinal lymph nodes were also identified (Figure 3). Magnetic resonance imaging (MRI) 
of the brain was significant for mass extension $4 \mathrm{~mm}$ into the right anterior cranial fossa. CT Chest, Abdomen, and Pelvis were notable for a large right breast mass as well as metastatic disease in the hilum, chest wall, lungs, liver, pancreas, peritoneal cavity, and right ovary.

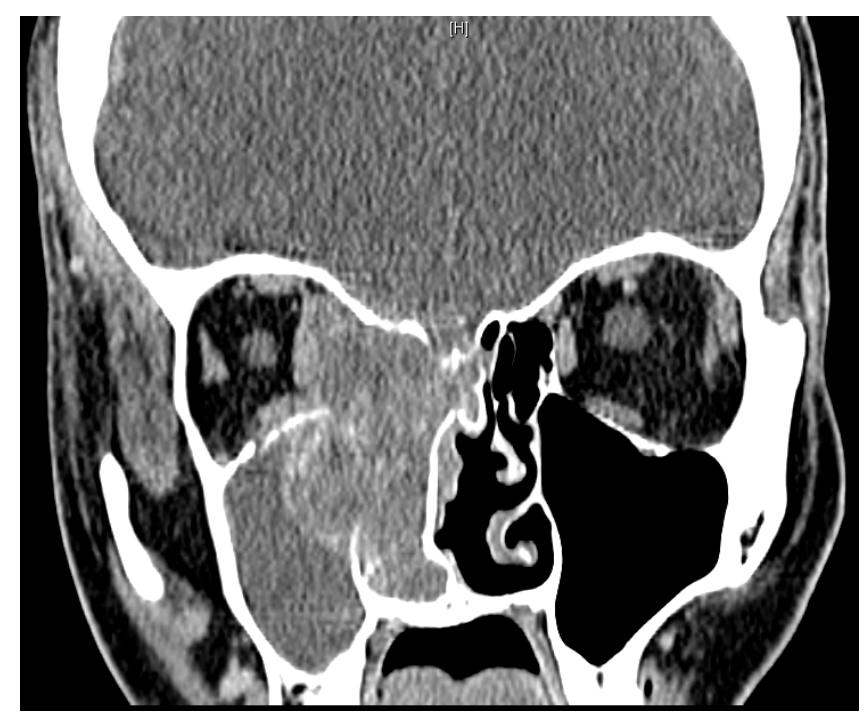

Figure 2. CT Sinus with Contrast on presentation to our hospital, coronal view. Large, heterogeneously enhancing mass of the right nasal cavity with post-obstructive opacificaiton of the right maxillary sinus. Bony destruction of the right lamina paprycea and cribiform plate.

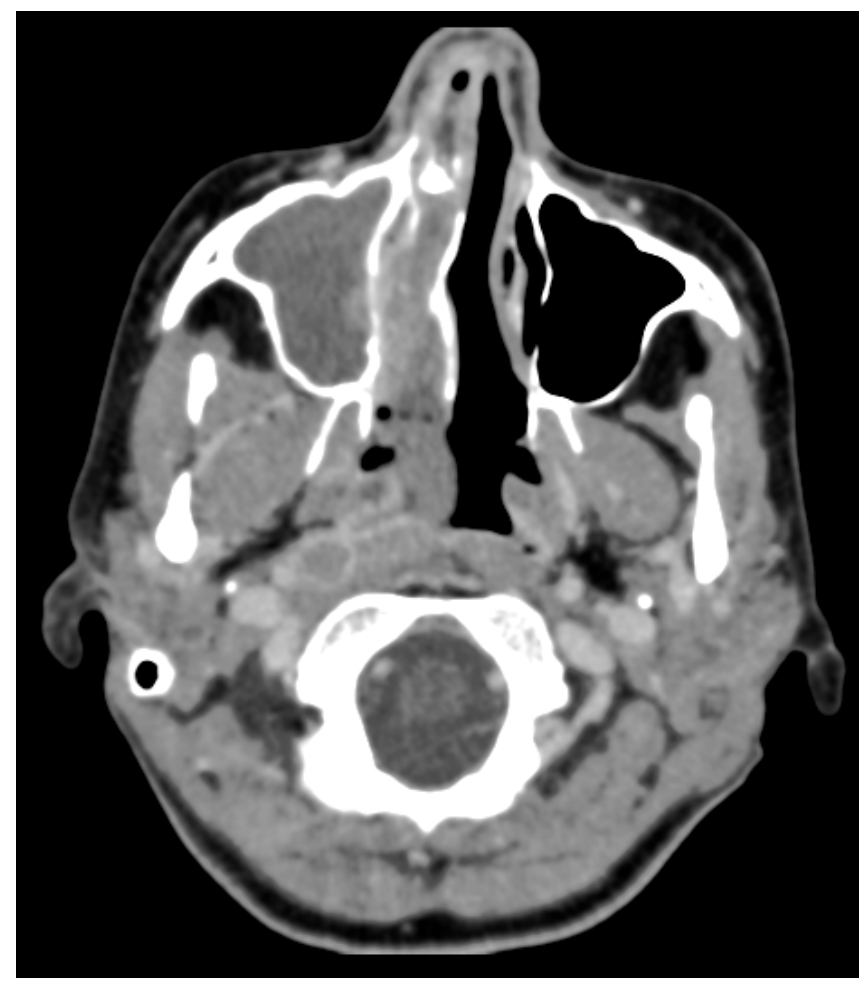

Figure 3. CT Sinus with Contrast on presentation to our hospital, axial view. Multiple enlarged pathologic-appearing lymph nodes seen in the right retropharyngeal space.

The decision was made to proceed to the operating room for endoscopic biopsy of the right nasal cavity mass and control of epistaxis. The right nasal cavity was notable for a friable mass that occupied the entire nasal cavity beyond the head of the inferior turbinate. Frozen sections were 
consistent with small blue cell neuroendocrine tumor, with similar morphology to the previously taken breast mass biopsy. A diagnosis of alveolar rhabdomyosarcoma was confirmed by pathology, which demonstrated the presence of a PAX3/7-FOXO1 fusion transcript (Figure 4).

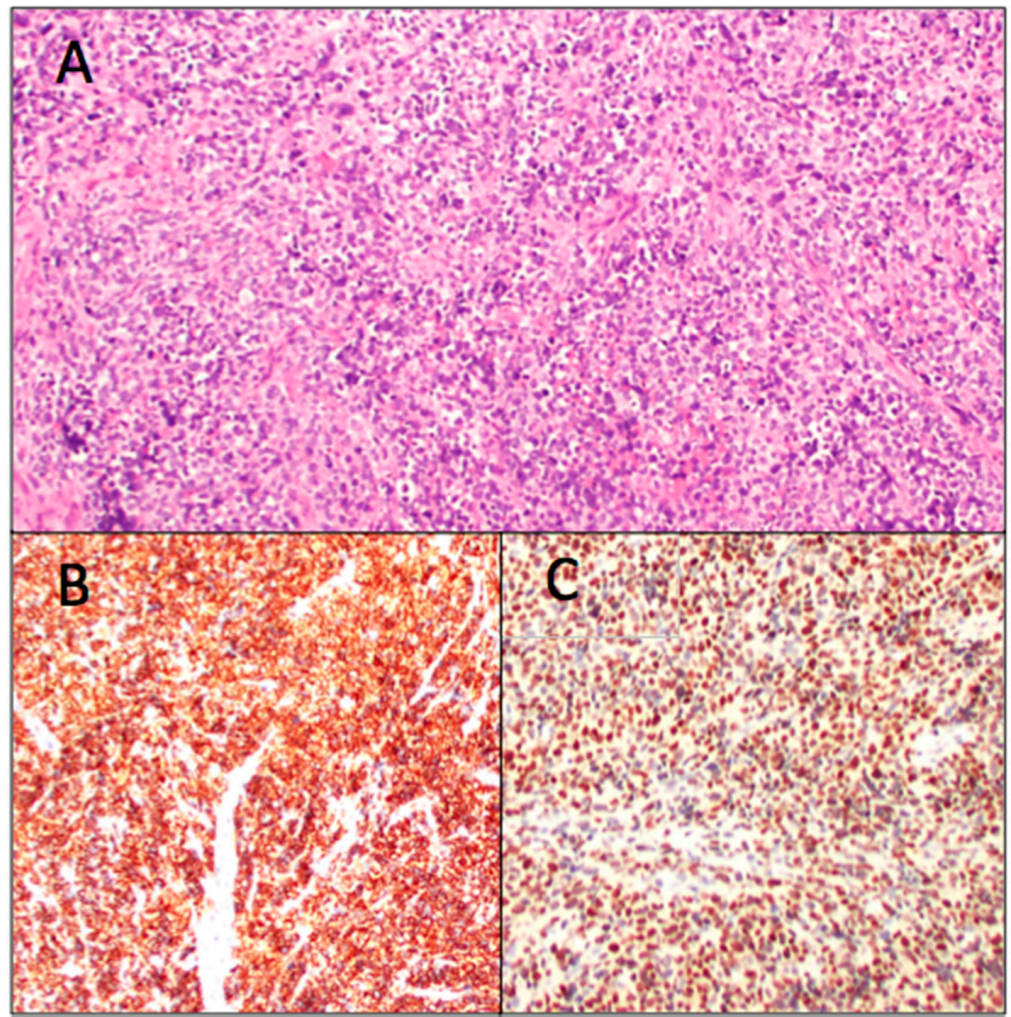

Figure 4. Pathology from nasal mass biopsy consistent with alveolar rhabdomyosarcoma. (A) Hematoxylin and eosin (H\&E) stain demonstrating a small round blue cell tumor. $(\mathbf{B}, \mathbf{C})$ Positive stain for desmin and myogen, respectively. Markers of skeletal muscle differentiation.

Final pathological review and tumor board discussion concluded that the patient's findings were consistent with widely metastatic alveolar rhabdomyosarcoma of the nasal cavity. She elected to pursue palliative chemotherapy, consisting of Vincristine/Dactinomycin/Cyclophosphomide. She initially responded well, but developed leptomeningeal metastases after cycle five. She completed palliative whole brain and sinus-directed radiation. Her neurologic symptoms progressed, and approximately six months after her biopsies, she transitioned to comfort care only.

\section{Discussion}

Rhabdomyosarcoma (RMS) is a rare, aggressive soft tissue malignancy that commonly occurs in the head and neck, albeit significantly more so in children and adolescents than in adults. Given the relative rarity of this malignancy, many institutions have limited experience in treating RMS. As such, although the relative survival of standard risk RMS patients has improved over the last 30 years, the prognosis for patients with relapsed and/or metastatic disease remains poor [8].

Among both adults and children, the most commonly encountered histopathological RMS subtype is embryonal, followed by alveolar, botryoid, and unclassified $[9,10]$. Alveolar RMS has been found to be the most unfavorable subtype in terms of both recurrence rate and overall survival [8,10]. Many large retrospective studies have found that pediatric patients with head and neck RMS have significantly better outcomes than adults, and this may be in part to the higher predominance of embryonal subtype in the pediatric population. 
Head and neck rhabdomyosarcoma can be further sub-classified by primary site: orbital, parameningeal, and non-orbital non-parameningeal. Parameningeal sites account for $41 \%-51.2 \%$ of head and neck RMS, and include the nasopharynx/nasal cavity, middle ear, paranasal sinuses, and the infratemporal fossa/pterygopalatine space [8,11]. Parameningeal RMS have been identified as unfavorable due to the fact that the lesions often invade critical anatomic structures, precluding complete resection [12]. Survival outcomes are significantly worse for parameningeal subsites; furthermore, there is a higher rate of direct meningeal extension, which is typically not found in patients with RMS of other sub-sites [13].

The development of distant metastases in patients with head and neck RMS is associated with significantly worse outcomes, specifically a $16 \%$ disease-specific survival at 5 years [9]. Although the specific locations and extent of distant disease is not quantified in the larger retrospective studies, prior case reports on adult head and neck RMS have only described metastatic disease to the lungs, bone, and liver. Here, we describe a patient with extensive distant metastases, including the breast, hilum, chest wall, lungs, liver, pancreas, peritoneal cavity, and right ovary. Of note, the finding of breast metastases in our patient is concordant with prior studies that have found RMS breast metastases only in female patients with alveolar subtype [14-16]. The extent of metastatic disease at the time of diagnosis raises the question of how impactful the month-long delay in diagnosis in terms of disease progression and overall likelihood of survival.

Although alveolar rhabdomyosarcoma is a rare cause of unilateral sinus opacification in adults, neoplasm in general should be considered in the setting of unilateral symptoms or radiologic findings. This is particularly true in cases of unilateral disease, where an anatomical etiology-such as a deviated septum, bony spur, or polyp-or odontogenic source is not identified on imaging or exam. Furthermore, neoplasm should be suspected in the setting of progressive disease that does not respond to traditional medical management. One retrospective study of 63 patients with unilateral sinus symptoms plus unilateral disease on $\mathrm{CT}$, who underwent sinus surgery, identified neoplasm as the cause of opacification in $15.4 \%$ of cases, with malignancy representing a smaller but still significant proportion of cases. Additionally, unilateral epistaxis—as was seen in our patient—was more frequently associated with neoplastic disease than benign causes such as chronic rhinosinusitis and nasal polyposis in that particular study population [17]. The existing literature on unilateral sinusitis and its association with underlying malignancy is limited and primarily focused on inverted papilloma, which has a rate of malignant degeneration to squamous cell carcinoma of between $3.4 \%$ [18] and 9.7\% [19]. The majority of other studies in this area focus specifically on unilateral maxillary sinusitis secondary to odontogenic sources. Nevertheless, given the potential for underlying malignancy in patients with unilateral sinus opacification on imaging, a comprehensive evaluation by an otolaryngologist including nasal endoscopy and consideration of imaging and biopsy should be considered. This is particularly true for patients like the one described here, with concerning signs or symptoms such as lack of anatomical cause for unilateral disease, progression of symptoms despite antibiotic management, and epistaxis.

\section{Conclusions}

Rhabdomyosarcoma of the paranasal sinuses is a rare malignancy, particularly in adults. Given the significantly worse outcomes in adults than children and the implications of distant metastases on survival, early diagnosis is of utmost importance. A high degree of clinical suspicion for neoplasm, including RMS, should be maintained when evaluating a patient with unilateral sinus disease.

Acknowledgments: No sources of funding were used in this study.

Author Contributions: Amanda E. Dilger, Alexander L. Schneider and Stephanie Shintani Smith were involved in the initial consultation, management and surgical procedure for the patient described in this case report. Amanda E. Dilger and John Cramer conceived the themes for this paper. Amanda E. Dilger, Alexander L. Schneider and John Cramer conducted the literature review and wrote this manuscript.

Conflicts of Interest: The authors declare no conflict of interest. 


\section{References}

1. Gonzalez-Gonzalez, R.; Bologna-Molina, R.; Molina-Frechero, N.; Domínguez-Malagon, H.R. Prognostic factors and treatment strategies for adult head and neck soft tissue sarcoma. Int. J. Oral Maxillofac. Surg. 2012, 41, 569-575. [CrossRef] [PubMed]

2. Chang, A.E.; Chai, X.; Pollack, S.M.; Loggers, E.; Rodler, E.; Dillon, J.; Parvathaneni, U.; Moe, K.S.; Futran, N.; Jones, R.L. Analysis of clinical prognostic factors for adult patients with head and neck sarcomas. Otolaryngol. Head Neck Surg. 2014, 151, 976-983. [CrossRef] [PubMed]

3. Tajudeen, B.A.; Fuller, J.; Lai, C.; Grogan, T.; Elashoff, D.; Abemayor, E.; John, M.S. Head and neck sarcomas: The UCLA experience. Am. J. Otolaryngol. 2014, 35, 476-481. [CrossRef] [PubMed]

4. Lindford, A.; McIntyre, B.; Marsh, R.; MacKinnon, C.A.; Davis, C.; Tan, S.T. Outcomes of the treatment of head and neck sarcomas in a tertiary referral center. Front. Surg. 2015, 2, 19. [CrossRef] [PubMed]

5. Peng, K.A.; Grogan, T.; Wang, M.B. Head and neck sarcomas: Analysis of the SEER database. Otolaryngol. Head Neck Surg. 2014, 151, 627-633. [CrossRef] [PubMed]

6. Ruiz-Mesa, C.; Goldberg, J.M.; Munoz, A.J.C.; Dumont, S.N.; Trent, J.C. Rhabdomyosarcoma in adults: New perspectives on therapy. Curr. Treat. Options Oncol. 2015, 16, 27. [CrossRef] [PubMed]

7. Ahmed, A.A.; Tsokos, M. Sinonasal rhabdomyosarcoma in children and young adults. Int. J. Surg. Pathol. 2007, 15, 160-165. [CrossRef] [PubMed]

8. Turner, J.H.; Richmon, J.D. Head and neck rhabdomyosarcoma: A critical analysis of population-based incidence and survival data. Otolaryngol. Head Neck Surg. 2011, 145, 967-973. [CrossRef] [PubMed]

9. Callender, T.A.; Weber, R.S.; Janjan, N.; Benjamin, R.; Zaher, M.; Wolf, P.; El-Naggar, A. Rhabdomyosarcoma of the nose and paranasal sinuses in adults and children. Otolaryngol. Head Neck Surg. 1995, 112, 252-257. [CrossRef]

10. Thompson, C.F.; Kim, B.J.; Lai, C.; Grogan, T.; Elashoff, D.; St John, M.A.; Wang, M.B. Sinonasal rhabdomyosarcoma: Prognostic factors and treatment outcomes. Int. Forum Allergy Rhinol. 2013, 3, 678-683. [CrossRef] [PubMed]

11. Wharam, M.D., Jr. Rhabdomyosarcoma of Parameningeal Sites. Semin. Radiat. Oncol. 1997, 7, 212-216. [CrossRef]

12. Douglas, J.G.; Arndt, C.A.; Hawkins, D.S. Delayed radiotherapy following dose intensive chemotherapy for parameningeal rhabdomyosarcoma (PM-RMS) of childhood. Eur. J. Cancer 2007, 43, 1045-1050. [CrossRef] [PubMed]

13. Tefft, M.; Fernandez, C.; Donaldson, M.; Newton, W.; Moon, T.E. Incidence of meningeal involvement by rhabdomyosarcoma of the head and neck in children: A report of the Intergroup Rhabdomyosarcoma Study (IRS). Cancer 1978, 42, 253-258. [CrossRef]

14. Persic, M.; Roberts, J.T. Alveolar rhabdomyosarcoma metastatic to the breast: Long-term survivor. Clin. Oncol. 1999, 11, 417-418. [CrossRef]

15. Audino, A.N.; Setty, B.A.; Yeager, N.D. Rhabdomyosarcoma of the breast in adolescent and young adult (AYA) women. J. Pediatr. Hematol. Oncol. 2017, 39, 62-66. [CrossRef] [PubMed]

16. Kebudi, R.; Koc, B.S.; Gorgun, O.; Celik, A.; Kebudi, A.; Darendeliler, E. Breast metastases in children and adolescents with rhabdomyosarcoma: A large single-institution experience and literature review. J. Peadiatr. Hematol. Oncol. 2017, 39, 67-71. [CrossRef] [PubMed]

17. Habesoglu, T.E.; Habesoglu, M.; Surmeli, M.; Uresin, T.; Egeli, E. Unilateral sinonasal symptoms. J. Craniofac. Surg. 2010, 21, 2019-2022. [CrossRef] [PubMed]

18. Pasquini, E.; Sciarretta, V.; Farneti, G.; Modugno, G.C.; Ceroni, A.R. Inverted papilloma: Report of 89 cases. Am. J. Otolaryngol. 2004, 25, 178-185. [CrossRef] [PubMed]

19. Yoskovitch, A.; Braverman, I.; Nachtigal, D.; Frenkiel, S. Sinonasal schneiderian papilloma. J. Otolaryngol. 1998, 27, 122-126. [PubMed]

(C) 2017 by the authors; licensee MDPI, Basel, Switzerland. This article is an open access article distributed under the terms and conditions of the Creative Commons Attribution (CC BY) license (http:/ / creativecommons.org/licenses/by/4.0/). 